\section{(2) OPEN ACCESS}

\title{
Patterns of atrophy in pathologically confirmed dementias: a voxelwise analysis
}

\author{
Lorna Harper, ${ }^{1}$ Femke Bouwman, ${ }^{2}$ Emma J Burton, ${ }^{3}$ Frederik Barkhof, ${ }_{1}^{4,5,6}$ \\ Philip Scheltens, ${ }^{2}$ John T O'Brien, ${ }^{7}$ Nick C Fox, ${ }_{1}^{1}$ Gerard R Ridgway, ${ }^{8,9}$ \\ Jonathan M Schott ${ }^{1}$
}

\begin{abstract}
- Additional material is published online only. To view please visit the journal online (http://dx.doi.org/10.1136/ jnnp-2016-314978).
\end{abstract}

For numbered affiliations see end of article.

Correspondence to Lorna Harper, Dementia Research Centre, ION UCL, 8-11 Queen Square, London WC1N 3BG, UK; lornaharper01@gmail. com

\section{GRR and JMS contributed} equally.

Received 26 September 2016 Revised 23 January 2017 Accepted 8 March 2017 Published Online First 4 May 2017

\section{SLinked}

- http://dx.doi.org/10.1136/ jnnp-2017-316016

\section{CrossMark}

To cite: Harper $L$, Bouwman F, Burton EJ, et al. J Neurol Neurosurg Psychiatry 2017:88:908-916.

\section{ABSTRACT}

Objective Imaging is recommended to support the clinical diagnoses of dementias, yet imaging research studies rarely have pathological confirmation of disease. This study aims to characterise patterns of brain volume loss in six primary pathologies compared with controls and to each other.

Methods One hundred and eighty-six patients with a clinical diagnosis of dementia and histopathological confirmation of underlying pathology, and 73 healthy controls were included in this study. Voxel-based morphometry, based on ante-mortem T1-weighted MRI, was used to identify cross-sectional group differences in brain volume.

Results Early-onset and late-onset Alzheimer's disease exhibited different patterns of grey matter volume loss, with more extensive temporoparietal involvement in the early-onset group, and more focal medial temporal lobe loss in the late-onset group. The Presenilin-1 group had similar parietal involvement to the early-onset group with localised volume loss in the thalamus, medial temporal lobe and temporal neocortex. Lewy body pathology was associated with less extensive volume loss than the other pathologies, although precentral/postcentral gyri volume was reduced in comparison with other pathological groups. Tau and TDP43A pathologies demonstrated similar patterns of frontotemporal volume loss, although less extensive on the right in the 4-repeat-tau group, with greater parietal involvement in the TDP43A group. The TDP43C group demonstrated greater left anteriortemporal involvement.

Conclusions Pathologically distinct dementias exhibit characteristic patterns of regional volume loss compared with controls and other dementias. Voxelwise differences identified in these cohorts highlight imaging signatures that may aid in the differentiation of dementia subtypes during life. The results of this study are available for further examination via NeuroVault (http://neurovault. org/collections/ADHMHOPN/).

\section{INTRODUCTION}

Cerebral atrophy is a downstream effect of neurodegeneration, ${ }^{1}$ with detectable changes in early ${ }^{2}$ and even presymptomatic ${ }^{3}$ disease stages. Patterns of cross-sectional atrophy are included as core or supporting features in many consensus diagnostic guidelines, ${ }^{4-8}$ and longitudinal atrophy rates are used as outcome measures in clinical trials. ${ }^{9}$ While there is substantial evidence to support the use of patterns of brain volume loss to aid in the differential diagnosis of dementia, ${ }^{10} 11$ much of the evidence base is derived from patients with clinical diagnoses, which may be inaccurate in up to $30 \%$ of cases, even in specialist centres. ${ }^{12}$ Identifying patterns of atrophy that help to distinguish pathological processes from each other (and from normal ageing), based on gold standard histopathology, may provide greater understanding of the ability of clinical imaging to predict dementia pathology and help to translate imaging features, identified through research, into clinically useful biomarkers.

To date, relatively few imaging studies using voxelwise approaches are based on cases with histopathological confirmation of diagnosis, and those who have typically used small sample sizes. Furthermore, many studies have made comparisons only between people with dementia and healthy controls (see table 1 for a review of the literature), whereas in clinical practice, structural imaging is increasingly used to distinguish between the different pathological forms of dementia. Motivated by this clinical question, we performed a comprehensive computational analysis of brain volume loss in the largest series yet reported, including 186 individuals with a diagnosis of dementia during life and primary histopathological diagnosis of one of six pathologies (Alzheimer's disease (AD), dementia with Lewy bodies (DLB), 3-repeat-tau, 4-repeat-tau, TDP43A and TDP43C (transactive response DNA-binding protein 43)). The aim of this study was to identify patterns of atrophy that would not only differentiate these primary neurodegenerative pathology groups from cognitively healthy control subjects but, critically, also from one another.

\section{MATERIALS AND METHODS}

\section{Study population}

Two hundred and fifteen people were identified with a usable T1-weighted MRI, a diagnosis of dementia during life and post-mortem $(n=206)$ or biopsy $(n=9)$ confirmation of the underlying pathology. Twenty-one individuals were excluded for having incomplete or inconclusive pathology, and eight individuals were excluded due to insufficient data to study them as a group ( $n=4$ amyloid precursor protein mutation carriers, $n=1$ TDP43B, $n=3$ with fused in sarcoma pathology). A total of 186 individuals were included in the subsequent analysis: 107 had a primary pathology diagnosis of AD (68 early-onset ( $<65$ years at disease onset), 29 lateonset ( $\geq 65$ years at disease onset), 10 presenilin-1 
Table 1 Voxel-based morphometry studies in pathology-confirmed (or genetically confirmed) dementias

\begin{tabular}{|c|c|c|c|c|c|c|}
\hline Study & Year & Defined pathologies & $\begin{array}{l}\text { Pathology confirmed } \\
\text { cases, } \mathrm{n}\end{array}$ & $\begin{array}{l}\text { Comparison with } \\
\text { controls }\end{array}$ & $\begin{array}{l}\text { Direct pathology } \\
\text { comparison }\end{array}$ & PubMed ID \\
\hline Whitwell et al & 2004 & Tau-positive FTLD, tau-negative FTLD & 9,8 & Yes & No & 16908994 \\
\hline Whitwell et al & 2005 & FTLD-U, Pick's, MAPT & $9,7,5$ & Yes & Yes & 16157747 \\
\hline Josephs et al & 2006 & $\begin{array}{l}\text { NA—various pathologies grouped by language } \\
\text { impairment features }\end{array}$ & 12 & NA & NA & 16613895 \\
\hline Grossman et al & 2007 & $\begin{array}{l}\text { Tau-positive FTLD, tau-negative FTLD, (frontal } \\
\text { variant)-AD }\end{array}$ & $5,4,3$ & Yes & No & 17998442 \\
\hline Josephs et al & 2008a & (aphasic)-AD, (aphasic)-FTLD-U, (typical)-AD & $5,5,10$ & Yes & Yes & 18166704 \\
\hline Josephs et al & $2008 b$ & PSP, CBD & 13,11 & Yes & No & 17097770 \\
\hline Josephs et al & $2008 c$ & Argyrophilic grain pathology & 12 & Yes & No & 17188783 \\
\hline Whitwell et al & 2008 & Braak stages III-VI & $10,13,32,27$ & Yes & NA & 18765650 \\
\hline Josephs et al & 2009 & $\begin{array}{l}\text { NA-FTLD groups defined based on clinical } \\
\text { features }\end{array}$ & 11 & NA & NA & 19884571 \\
\hline Pereira et al & 2009 & Tau-positive FTLD, FTLD-U & 6,9 & Yes & No & 19433738 \\
\hline Hu et al & 2010 & $\begin{array}{l}\text { NA—various pathologies grouped by language } \\
\text { impairment features }\end{array}$ & 8 & NA & NA & 20713948 \\
\hline Josephs et al & 2010 & (CBS)-CBD, (CBS)-AD & 6,5 & Yes & Yes & 20629131 \\
\hline Rohrer et al & 2010a & GRN, MAPT & 9,11 & Yes & Yes & 20045477 \\
\hline Rohrer et al & $2010 \mathrm{~b}$ & FTLD-TDP43 (types A-C and unspecified) & $9,5,10,4$ & Yes & No & 21172843 \\
\hline Whitwell et al & 2010a & (CBS)-TDP43, (CBS)-AD, (CBS)-CBD, (CBS)-PSP & $5,6,7,6$ & Yes & Yes & 21098403 \\
\hline Whitwell et al & 2010b & FTLD-TDP43 (types A-C) & $22,9,11$ & Yes & Yes & 21172844 \\
\hline Lee et al & 2011 & $\begin{array}{l}\text { CBD, (CBS)-AD, (CBS)-CBD, (CBS)-PSP, (CBS)-TDP, } \\
\text { (CBS)-mixed }\end{array}$ & $18,9,14,5,5,5$ & Yes & Yes & 21823158 \\
\hline Rankin et al & 2011 & (bvFTD)-CBD, (bvFTD)-Pick's & 5,5 & Yes & Yes & 21881831 \\
\hline Rohrer et al & 2011 & Pick's, MAPT, CBD, TDP43A, TDP43C & $9,6,5,6,12$ & Yes & No & 21908872 \\
\hline Whitwell et al & 2011a & (typical)-AD, (atypical)-AD, FTLD & $14,14,14$ & Yes & Yes & 19914744 \\
\hline Whitwell et al & $2011 b$ & Pick's, CBD, TDP43A & $5,5,5$ & Yes & Yes & 21556732 \\
\hline Boxer et al & 2012 & $\begin{array}{l}\text { NA-FTLD and AD correlations with saccade } \\
\text { parameters }\end{array}$ & 37 & NA & NA & 22491196 \\
\hline Hornberger et al & 2012 & (bvFTD)-FTLD, AD & 19,18 & Yes & No & 23012333 \\
\hline Khan et al & 2012 & (slowly progressive bvFTD)-C9ORF72 & 2 & Yes & No & 22399793 \\
\hline Mahoney et al & 2012 & C9ORF72, MAPT, GRN & $11,11,8$ & Yes & Yes & 22366791 \\
\hline Whitwell et al & 2012a & $\begin{array}{l}\text { NA_bvFTD groups defined based on frontal } \\
\text { lobe symmetry }\end{array}$ & 80 & NA & NA & 22502999 \\
\hline Whitwell et al & $2012 b$ & MAPT, GRN, C9ORF72, FTLD & $25,12,19,12$ & Yes & Yes & 22366795 \\
\hline Whitwell et al & $2012 c$ & $\begin{array}{l}\text { typical } A D \text {, hippocampal-sparing } A D \text {, limbic- } \\
\text { predominant } A D\end{array}$ & $125,19,33$ & Yes & Yes & 22951070 \\
\hline Cash, Ridgway et al & 2013 & $\begin{array}{l}\text { (presymptomatic)-familial AD, (symptomatic)- } \\
\text { familial AD }\end{array}$ & 69,50 & Yes & Yes & 24049139 \\
\hline Caso et al & 2013 & Pick's & 1 & Yes & No & 22713404 \\
\hline Coon et al & 2013 & FTLD-MND & 2 & Yes & No & 22051030 \\
\hline Scahill et al & 2013 & APP, PSEN1 & 10,18 & Yes & Yes & 23380992 \\
\hline Toledo et al & 2013 & $A D, A D+M T L, A D+D L B, A D+D L B+M T L$ & $7,5,6,4$ & No & Yes & 24252435 \\
\hline Josephs et al & 2013a & (typical)-AD, (IvPPA)-AD & 20,10 & Yes & No & 23541297 \\
\hline Josephs et al & $2013 b$ & $\begin{array}{l}\text { CSTD-positive TDPC, CSTD-equivocal TDPC, } \\
\text { CSTD-negative TDPC }\end{array}$ & $2,5,5$ & Yes & Yes & 23358603 \\
\hline Murray et al & 2013 & $\begin{array}{l}\text { NA-DLB groups defined by presence of REM } \\
\text { sleep behaviour disorder }\end{array}$ & 75 & NA & NA & 24107861 \\
\hline Caso et al & 2014 & (nfvPPA)-tau, (nfvPPA)-TDP43 & 9,2 & Yes & No & 24353332 \\
\hline Henry et al & 2014 & TDP43C & 1 & Yes & No & 23171151 \\
\hline Josephs & 2014 & TDP43-positive AD, TDP43-negative AD & 195,147 & Yes & Yes & 24659241 \\
\hline Nedelska et al & 2015 & DLB, DLB-AD, AD & $20,22,30$ & Yes & Yes & 25128280 \\
\hline Ossenkoppele et al & 2015 & $\begin{array}{l}\text { (behavioural/dysexecutive)-AD, (typical)-AD, } \\
\text { (bvFTD)-FTLD }\end{array}$ & $24,17,12,8,21$ & Yes & Yes & 26141491 \\
\hline Sala-Llonch et al & 2015 & Symptomatic-PSEN1, asymptomatic-PSEN1 & 11,13 & Yes & No & 25638532 \\
\hline Shingawa et al & 2015 & $\begin{array}{l}\text { NA-C9ORF72 groups were defined based on } \\
\text { the presence of delusions }\end{array}$ & 17 & NA & NA & 25342578 \\
\hline
\end{tabular}

Parentheses indicate additional clinical features or diagnosis. The number of pathology cases is listed per defined pathology group.

AD, Alzheimer's disease; APP, amyloid precursor protein mutation carriers; bvFTD, behavioural variant FTD; C9ORF72, chromosome 9 open reading frame 72 mutation carriers; CBD, corticobasal degeneration; CBS, corticobasal syndrome; CTSD, corticospinal tract degeneration; DLB, dementia with Lewy bodies; FTD, frontotemporal dementia; FTLD, frontotemporal lobar degeneration; FTLD-MND, FTLD with motor neuron disease; FTLD-U, FTLD with ubiquitin-positive (tau-synuclein-negative and alpha-synuclein-negative) inclusions; GRN, progranulin mutation carriers; MAPT, microtubule-associated protein tau mutation carriers; MTL, medial temporal lobe pathology; NA, not applicable; nfvPPA, non-fluent variant primary progressive aphasia; PSEN1, presenilin-1 mutation carriers; PSP, progressive supranuclear palsy; TDP43, transactive response DNA-binding protein 43. 
mutation carriers), 25 with DLB, 11 with 3-repeat-tau, 17 with 4-repeat-tau, 12 TDP43A, and 14 TDP43C. Pathological examination of brain tissue was carried out between 1997 and 2015 according to standard histopathological processes and criteria in use at the time of assessment at one of four centres: the Queen Square Brain Bank, London; Kings College Hospital, London; VU Medical Centre, Amsterdam and Institute for Ageing and Health, Newcastle. The study included 73 cognitively normal control subjects (based on clinical diagnosis) who were recruited to various imaging studies that had been carried out at the Dementia Research Centre, London. Controls were separated into younger ( $<65$ years of age at the time of scan, $n=33$ ) and older ( $\geq 65$ years of age at the time of scan, $n=40$ ) groups to better match the patient groups. All patients or their legal representatives consented, during life, to brain donation and use of their clinical data for research purposes at each of the participating sites. Ethical approval for this retrospective study was obtained from the National Research Ethics Service Committee London-South East. Group demographics were compared using Kruskal-Wallis tests, with a significant result being followed by post hoc pairwise multiple comparisons testing using Dunn's test. A subset of these subjects was included in earlier work focusing on visual rating scales. ${ }^{13}$ Data processing and analyses were performed using Python libraries NumPy 1.8.1 (http:// www.numpy.org/), SciPy 0.14.0 (https://www.scipy.org/scipylib/ index.html) and Pandas 0.14.1 (http://pandas.pydata.org/) on Python 2.7.6-64-bit.

\section{MRI scanning}

All individuals had T1-weighted volumetric MRI performed during life. As the data were collected retrospectively from multiple centres, the images were acquired on scanners from three different manufacturers (Philips, GE, Siemens) using a variety of different imaging protocols. Magnetic field strength varied between $1.0 \mathrm{~T}(\mathrm{n}=15$ scans $), 1.5 \mathrm{~T}(\mathrm{n}=201$ scans) and $3 \mathrm{~T}$ ( $\mathrm{n}=43$ scans).

\section{Image analysis}

Voxel-based morphometry (VBM) preprocessing and analysis was performed using SPM12b (Statistical Parametric Mapping, V.12b revision 5829; http://www.fil.ion.ucl.ac.uk/spm) and Matlab V.R2012a (7.14.0.739-64-bit, uk.mathworks.com/products/matlab/). Due to the variability in scanning parameters, an initial rigid registration to the Montreal Neurological Institute International Consortium for Brain Mapping 152 (ICBM152) template was performed using the Reg-Aladin tool from the NiftyReg package (https://sourceforge.net/projects/niftyreg/) to provide a better starting point for the SPM preprocessing pipeline. Each registration was then checked and manually adjusted (if necessary) such that the anterior commissure was within a few millimetres of the origin and the orientation was within a few degrees of the ICBM152 template. Grey matter, white matter and cerebrospinal fluid (CSF) segmentations were obtained using the unified segmentation approach ${ }^{14}$ with default settings. A group average tissue probability map was generated through iterative alignment of the initial grey and white matter segmentations to an evolving estimate of their groupwise average using the Dartel toolbox. ${ }^{15}$ The initial grey and white matter segmentations were then warped using the Dartel transformations and modulated to account for local volume changes, then smoothed with a $6 \mathrm{~mm}$ full width at half maximum Gaussian kernel. Grey and white matter masks were created based on the optimal threshold of the group average segmentations using the automatic mask creation strategy in the SPM Masking toolbox (http://www0.cs.ucl.ac.uk/ staff/g.ridgway/masking/).

The imaging data were assessed by applying the general linear model at the level of each voxel using all images $(n=259)$ and modelling the following terms: a 10-level group factor (younger controls, older controls, early-onset AD, presenilin-1 mutation carriers, late-onset $\mathrm{AD}, \mathrm{DLB}$, 3-repeat-tau, 4-repeat-tau, TDP43A and TDP43C); factors representing magnetic field strength, imaging site and sex; and covariates for age at the time of scanning and total intracranial volume (computed by summing up probabilistic voxel volumes in grey matter, white matter and CSF segmentations). The model was used to investigate the pairwise contrasts between the primary pathology groups, with the AD pathology group stratified by age at disease onset and genetic mutation status. The groups were also contrasted with age-matched controls (ie, older controls were contrasted with the late-onset $\mathrm{AD}$ and DLB groups; younger controls were contrasted with all other groups). Correction for multiple comparisons was made using random field theory (for peak height) to control the familywise error (FWE) rate at a significance level of $\mathrm{p}<0.001$, or 0.05 in most cases, although some results are shown at an uncorrected level of $\mathrm{p}<0.001$. Unthresholded effect size maps are also displayed to allow better characterisation of brain volume loss that did not reach statistical significance.

\section{RESULTS}

\section{Study population}

Demographic details of the patients and control subjects are presented in table 2. As expected, the late-onset AD group and the DLB group were, on average, older than the other groups at disease onset, and therefore, at the time of scanning, although this did not reach statistical significance in all cases (see online supplementary table 1). Between-group testing of time from scan until death was statistically significant $(\mathrm{p}<0.05)$ with the DLB group having the shortest mean interval (3.7 years) and the TDP43C group with the longest mean interval (7.0 years); however, these differences did not survive multiple comparisons correction. There were no statistically significant differences in sex $(p=0.05)$, disease duration $(p=0.13)$, Mini-Mental State Examination within 12 months of scanning $(p=0.24)$ or total intracranial volume $(\mathrm{p}=0.20)$ between the pathology groups.

\section{Comparison with age-matched controls}

Using a strict statistical threshold $(\mathrm{p}<0.001 \mathrm{FWE})$, significant differences in grey matter volume were found between each of the pathology groups and the appropriate age-matched control group (figure 1). The early-onset AD group demonstrated a diffuse pattern of atrophy, predominantly affecting the parietal and temporal cortex, with some additional extension into the frontal lobes. The presenilin-1 subgroup had similar parietal extension with more localised volume loss in the thalamus, medial temporal lobe and temporal neocortex. Compared with older control subjects, the late-onset AD group had a more focal pattern of grey matter volume loss in the medial temporal lobes, particularly the hippocampi. The DLB group also demonstrated significant medial temporal lobe atrophy when compared with older controls; however, this was much less extensive than the changes seen in the AD group, affecting the amygdalae (bilaterally, although worse on the left), the region of the left choroid fissure and a very small region around the lateral superior temporal gyrus. Relaxing the statistical threshold to $\mathrm{p}<0.05 \mathrm{FWE}$ (figure 2), further volume 


\begin{tabular}{|c|c|c|c|c|c|c|c|c|c|c|}
\hline & $\begin{array}{l}\text { Younger } \\
\text { controls }\end{array}$ & $\begin{array}{l}\text { Older } \\
\text { controls }\end{array}$ & EOAD & PSEN1 & LOAD & DLB & 3R-tau & 4R-tau & TDP43A & TDP43C \\
\hline $\mathrm{N}$ & 33 & 40 & 68 & 10 & 29 & 25 & 11 & 17 & 12 & 14 \\
\hline Sex (\%male) & 30 & 70 & 65 & 60 & 66 & 72 & 72 & 53 & 50 & 50 \\
\hline Age at onset (years) & NA & NA & $55.3(5.7)$ & $39.6(4.6)$ & $71.7(6.0)$ & $67.1(6.1)$ & $57.2(6.3)$ & $61.4(9.7)$ & $58.2(6.5)$ & $61.6(8.0)$ \\
\hline Age at scan (years) & $59.9(4.8)$ & $72.2(5.2)$ & $60.5(6.2)$ & $44.0(4.2)$ & $75.8(5.7)$ & $71.3(6.0)$ & $62.6(6.2)$ & $65.7(9.3)$ & $60.3(7.7)$ & $66.0(6.6)$ \\
\hline $\begin{array}{l}\text { Disease duration } \\
\text { at scan (years) }\end{array}$ & NA & NA & $5.2(2.9)$ & $4.4(2.3)$ & $4.1(2.1)$ & $4.2(2.9)$ & $5.4(2.1)$ & $4.4(2.4)$ & $2.9(2.4)$ & $4.5(3.0)$ \\
\hline MMSE $(\times / 30)$ & NA & NA & $18(7)$ & $15(6)$ & $19(5)$ & $20(5)$ & $21(7)$ & $23(5)$ & $17(8)$ & $23(6)$ \\
\hline $\begin{array}{l}\text { Time from scan } \\
\text { until death (years) }\end{array}$ & NA & NA & $5.8(2.9)$ & $4.2(2.2)$ & $5.7(3.3)$ & $3.7(2.3)$ & $6.0(3.5)$ & $4.8(2.3)$ & $5.2(4.0)$ & $7.0(3.4)$ \\
\hline TIV (mL) & 1425 (121) & 1531 (156) & 1504 (155) & 1440 (163) & 1484 (136) & 1530 (149) & 1506 (164) & 1517 (178) & 1480 (133) & $1483(165)$ \\
\hline 1.5 T scans & $79 \%$ & $73 \%$ & $77 \%$ & $80 \%$ & $86 \%$ & $76 \%$ & $91 \%$ & $76 \%$ & $50 \%$ & $86 \%$ \\
\hline 3.0 T scans & $21 \%$ & $27 \%$ & $13 \%$ & $20 \%$ & $7 \%$ & $8 \%$ & $9 \%$ & $24 \%$ & $33 \%$ & $7 \%$ \\
\hline
\end{tabular}

Data are reported as mean (SD).

DLB, dementia with Lewy bodies; EOAD, early-onset Alzheimer's disease; LOAD, late-onset Alzheimer's disease; MMSE, Mini-Mental State Examination (within 12 months of scanning); NA, not applicable; PSEN1, presenilin-1 mutation carriers; TDP43, transactive response DNA-binding protein 43; TIV, total intracranial volume.

differences were seen in the region of the right amygdala and choroid fissure; however, extension into other temporal lobe regions was confined to the left hemisphere. The frontotemporal lobar degeneration (FTLD)-tau groups had extensive grey matter volume loss in the frontal lobe affecting the superior, middle and inferior frontal gyri, extending into the insula. However, the right hemisphere was less severely affected in the 4-repeat-tau group, whereas the 3-repeat-tau group showed greater extension into the anterior temporal lobes. The TDP43A group demonstrated a similar, more symmetrical pattern of frontal volume loss, with additional volume loss extending into parietal lobe regions. The thalamic region was significantly affected in all three FTLD pathologies (3-repeat-tau, 4-repeat-tau and TDP43A). The TDP43C group had a significant volume loss in the temporal lobe, particularly anteriorly and in the left hemisphere where it extended into the frontal piriform and insular cortex. The reverse contrast was also investigated in all groups but showed no statistically

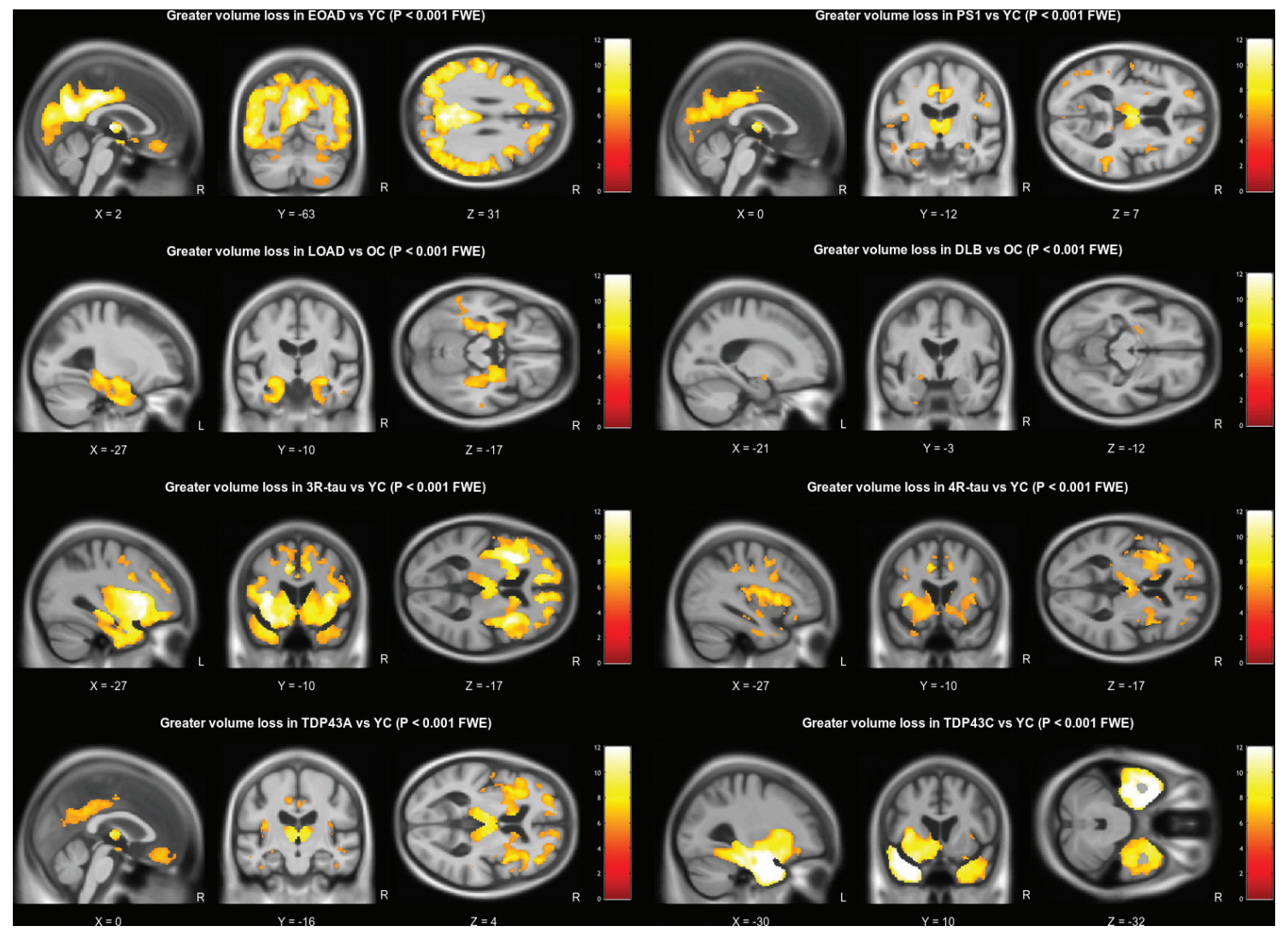

Figure 1 Grey matter volume differences based on pairwise comparison with the appropriate age-matched control group. All results are shown at a familywise error corrected significance level of $p<0.001$. Brain slices are displayed based on peak voxel location. DLB, dementia with Lewy bodies; EOAD, early-onset Alzheimer's disease; FTLD, frontotemporal lobar degeneration; L, left; LOAD, late-onset Alzheimer's disease; OC, older controls; R, right; YC, younger controls. 


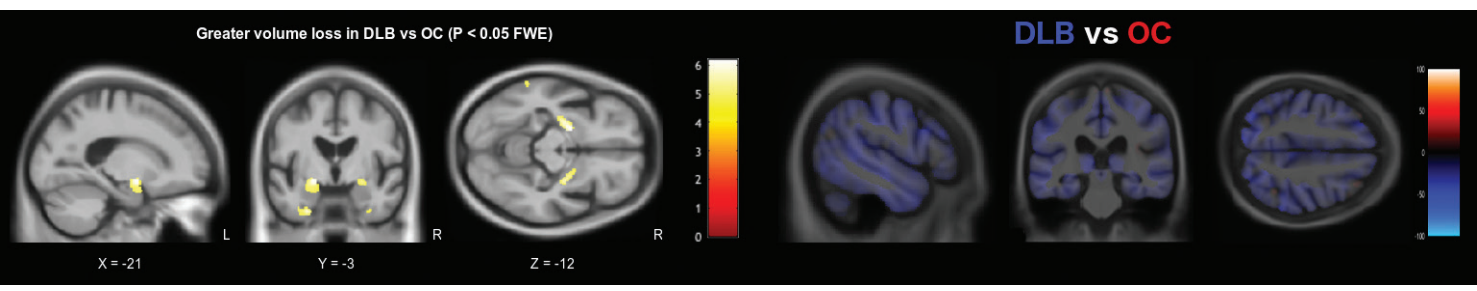

Figure 2 DLB versus older controls. Grey matter volume differences based on pairwise comparison between the DLB and older control groups. The statistical parametric map is shown on the left with brain slices displayed based on peak voxel location. Results are thresholded at a significance level of $p<0.05$ familywise error corrected. The axial effect size map is shown on the right, regions in blue represent greater volume loss in the DLB group. $D L B$, dementia with Lewy bodies; FWE, familywise error rate; L, left; OC, older controls; $R$, right.

significant regions where volume loss was more pronounced in the control group.

\section{Comparison between pathology groups}

Differences in grey matter volume based on pairwise comparisons of each of the pathology groups are presented in figures 3 and 4. The early-onset $\mathrm{AD}$ group had significant grey matter volume loss in parietal lobe regions $(p<0.05$ FWE) compared with the other groups (figure 2); however, in comparison with the presenilin-1 and TDP43A groups, the differences were very small and did not survive correction for multiple comparisons.

The presenilin-1 group had greater volume loss in the thalamus bilaterally compared with the early-onset AD group $(\mathrm{p}<0.001$ uncorrected $)$, and around the cingulate gyrus in comparison with both the early-onset and late-onset AD group $(p<0.05$ FWE $)$ and the DLB group $(p<0.05$ FWE). In comparison with the FTLD groups, greater volume loss was observed in more posterior brain regions, also around the cingulate gyrus when contrasted with the 3-repeat-tau $(p<0.05$ FWE) and TDP43C groups $(\mathrm{p}<0.05 \mathrm{FWE})$, and around the superior parietal lobule when contrasted with the 4-repeat-tau $(\mathrm{p}<0.05$ FWE) and TDP43A groups ( $\mathrm{p}<0.001$ uncorrected).

The late-onset AD group also demonstrated significant volume loss in parietal and occipital cortical regions in comparison with DLB, tau and TDP43C groups ( $\mathrm{p}<0.001$ uncorrected). In addition, the associated effect size maps also highlighted smaller hippocampal volumes in all but the 3-repeat-tau comparison and the left hippocampus when compared with the TDP43C pathology group.

On the basis of the effect size maps, the DLB group demonstrated greater volume loss in the region of the precentral and postcentral gyri, the precuneus and the cerebellum in comparison with all other groups; however, none of these differences remained after correction for multiple comparisons.

The FTLD pathology groups demonstrated greater and extensive volume loss in frontal and temporal lobe regions in comparison with all other groups (figures 3 and 4). The 3-repeat tau group had significantly smaller grey matter volume in the frontal cortex in comparison with all other pathology groups $(\mathrm{p}<0.05 \mathrm{FWE}$, figures 3 and 4$)$, particularly around the piriform and insular cortex, and around the anterior cingulate gyrus except in comparison with the 4-repeat-tau and TDP43A groups. The 4-repeat-tau group also had significant volume loss around the anterior cingulate, although this did not survive correction for multiple comparisons for all contrasts. The TDP43A group demonstrated greater volume loss around the lateral orbito-frontal and inferior-frontal gyrus in comparison with the DLB and TDP43C groups, while in comparison with the late-onset $\mathrm{AD}$ group, volume differences were localised to the left anterior striatum $(\mathrm{p}<0.05 \mathrm{FWE})$. As expected, the TDP43C group showed extensive grey matter volume loss in the left anterior temporal lobe in comparison with all other pathology groups, extending into the right anterior temporal lobe in comparison with the late-onset AD group, the DLB and the 4-repeat-tau groups $(\mathrm{p}<0.05 \mathrm{FWE})$.

While it is only possible to show a few representative brain slices in each figure (figures 3-5), unthresholded statistical maps have been uploaded to the NeuroVault repository (http:// neurovault.org/collections/ADHMHOPN/) to allow for further inspection of grey matter and white matter volume differences throughout the brain for each comparison.

\section{DISCUSSION}

To our knowledge, this is the largest VBM imaging study to investigate brain volume differences between different pathologically proven dementias, allowing for a comprehensive assessment of differences between pathology groups and healthy controls, as well as direct comparisons between pathology groups (see table 1 for a review of the existing literature). Care was taken to age-match the patient groups to controls in order to reduce the influence of age-related volume loss, and results were also statistically adjusted for age (at time of scan), sex and total intracranial volume, ${ }^{16}$ as well as MRI field strength and site. Individuals with $\mathrm{AD}$ pathology were also separated into autosomal-dominant presenilin-1 mutation carriers, early-onset (before 65 years) and late-onset groups to investigate patterns of atrophy reported in relation to these often quite distinct disease phenotypes. ${ }^{17} 18$ Individuals with FTLD-tau were stratified based on the predominant tau isoform found at post-mortem. In comparison with healthy controls, results are reported based on a strict statistical threshold, controlling the FWE rate at a significance level of $\mathrm{p}<0.001$ (ie, fewer than 1 in 1000 statistical maps would have any false-positive voxels).

$\mathrm{AD}$ pathology was associated with extensive volume loss, particularly in temporoparietal regions (figure 1), in keeping with the known locus of AD pathology. However, the earlyonset group demonstrated more extensive parietal lobe atrophy, whereas the late-onset group demonstrated more focal medial temporal lobe volume loss (figure 1). In the direct comparison between the two groups, only volume differences in the left parietal lobe were statistically significant. This confirms previous reports from smaller studies based on clinical diagnosis. ${ }^{19}$ This, in turn, has clinical implications, confirming that, despite research criteria that focus on the presence/absence of hippocampal atrophy for the diagnosis of $\mathrm{AD}$, this may not be a strong feature in patients with early-onset disease.

The late-onset AD group also demonstrated smaller parietal/ occipital lobe volumes in comparison with the DLB, FTLD-tau and TDP43C groups (figures 2 and 3), in keeping with reported disruption to the brain's default mode network due to $\mathrm{AD}$ 


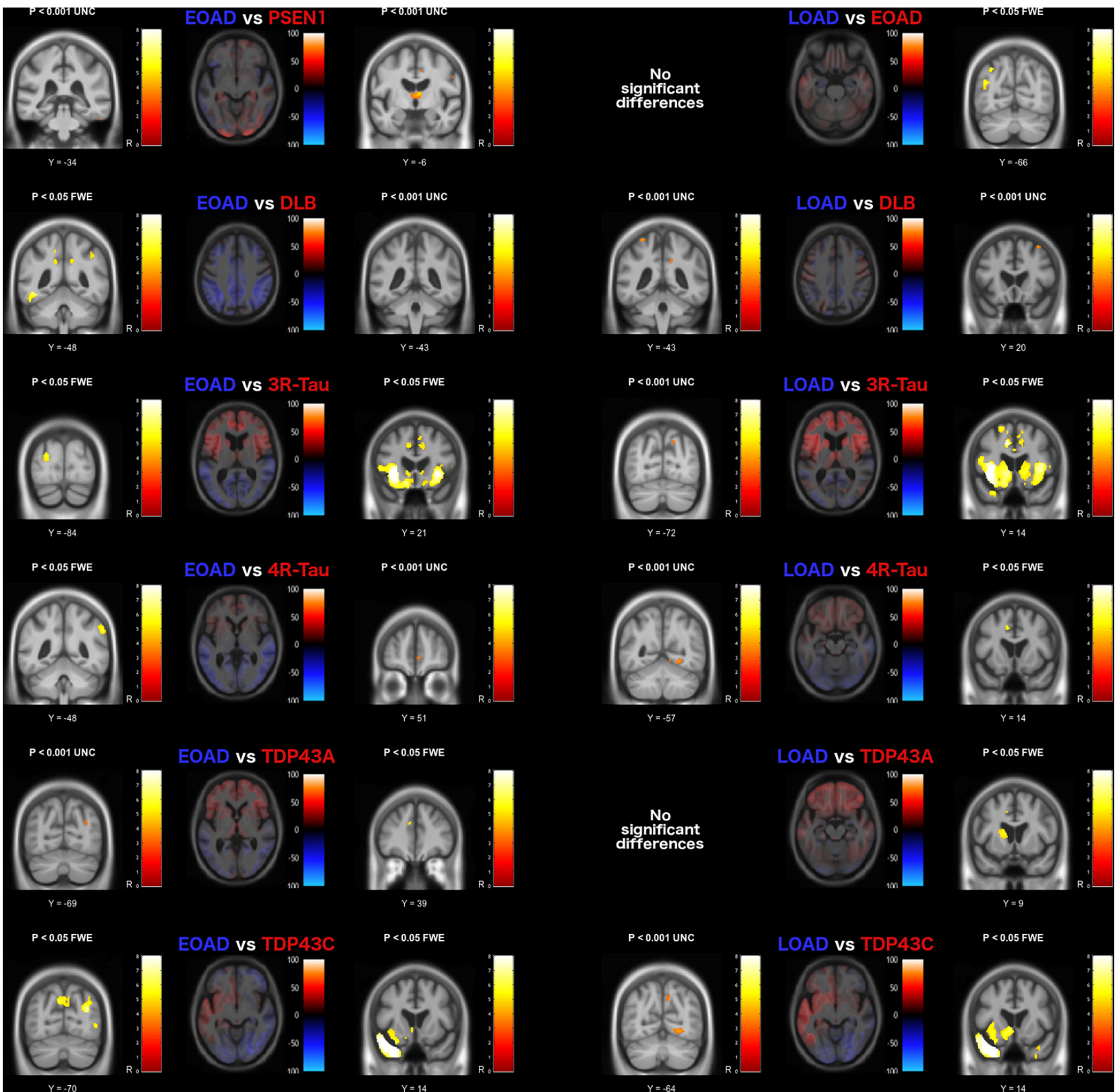

Figure 3 Grey matter volume differences based on pairwise comparison with the Alzheimer's disease pathology groups. The axial image slices are effect size maps and the label shown above each map indicates the group comparison. Text colour relates to image colour, with the coloured regions in the image representing greater volume loss in the associated group, and brain slices chosen to represent the most interesting findings. The statistical parametric map slices to the left and right of the effect size maps represent the forward and reverse contrasts associated with each group comparison, with brain slices displayed based on peak voxel location. Greater volume loss in the blue group is shown on the left, with the red group shown on the right statistical parametric map. DLB, dementia with Lewy bodies; EOAD, early-onset Alzheimer disease; FTLD, frontotemporal lobar degeneration; FWE, familywise error rate; L, left; LOAD, late-onset Alzheimer's disease; $R$, right; UNC, uncorrected (for multiple comparisons).

pathology. ${ }^{20}$ This was also evident in the other Alzheimer's pathology groups, with additional involvement of the anterior cingulate cortex.

In comparison with healthy controls and the early-onset AD group, the autosomal-dominant mutation carriers demonstrated focal volume loss in thalamic regions, which has recently been described as a potentially critical hub to explain the symptomology of the disease. ${ }^{21}$ Increased volume loss in this region has previously been reported in presymptomatic and symptomatic presenilin-1 mutation carriers compared with controls ${ }^{18} 22$ but not between presenilin-1 and sporadic early-onset AD as shown here.
Similar to the other neurodegenerative pathologies included in this study, patients with primary DLB pathology demonstrated global volume loss in comparison with the age-matched controls (see effect size map in figure 2). However, when contrasted directly with the other groups, the effect was much smaller, with relative sparing of the hippocampi, consistent with previous reports. ${ }^{23-26}$ Statistical significance was reached in the region of the amygdalae bilaterally. However, volume reduction in this region also correlates with neurofibrillary tangle burden, which may explain why significant differences were not seen in the direct comparison of DLB with AD. ${ }^{23} 27$ When compared with the other pathologies, DLB was associated with reduced grey 


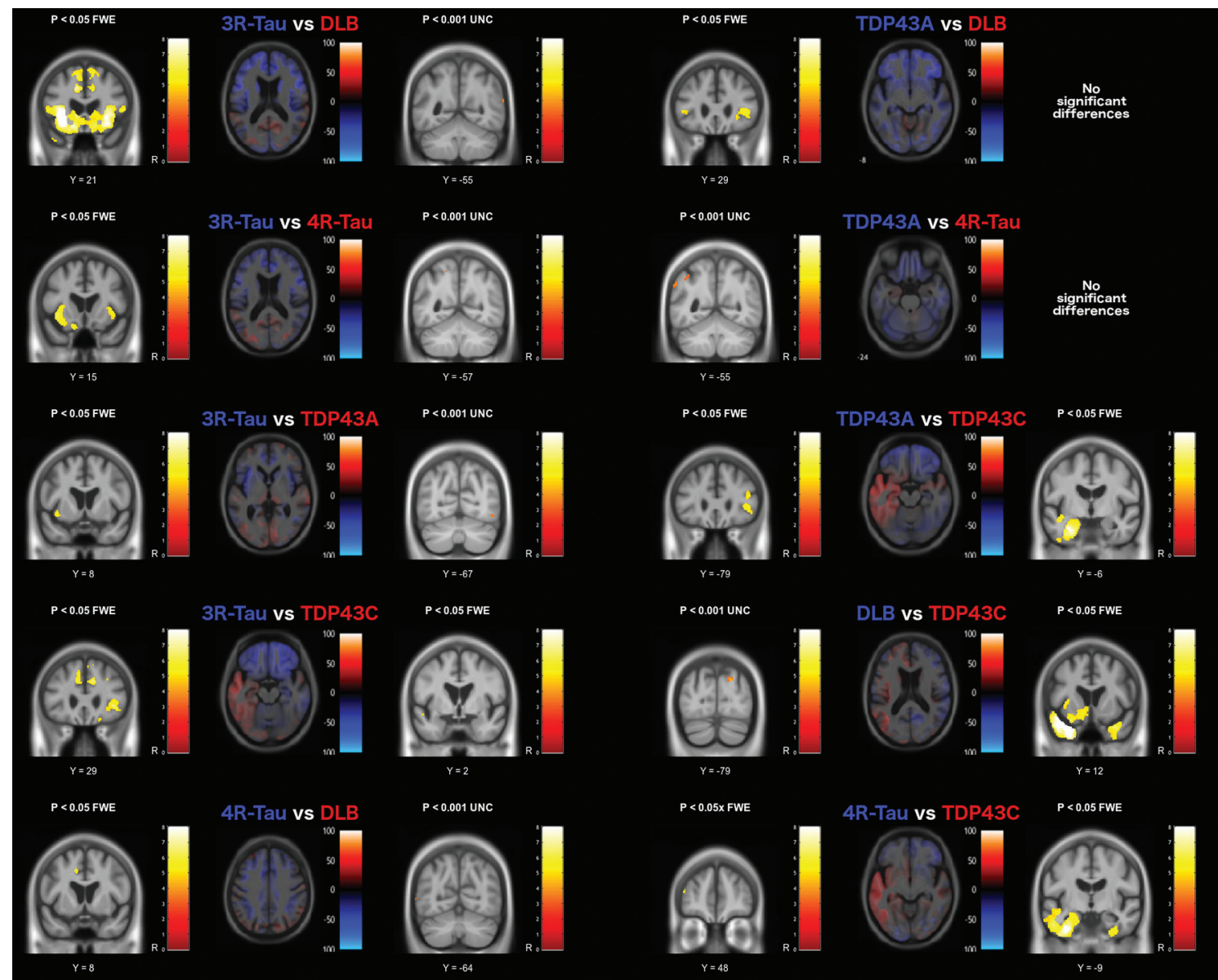

Figure 4 Grey matter volume differences based on pairwise comparison with the FTLD pathology groups. The axial image slices are effect size maps and the label shown above each map indicates the group comparison. Text colour relates to image colour, with the coloured regions in the image representing greater volume loss in the associated group, and brain slices chosen to represent the most interesting findings. The statistical parametric map slices to the left and right of the effect size maps represent the forward and reverse contrasts associated with each group comparison, with brain slices displayed based on peak voxel location. Greater volume loss in the blue group is shown on the left, with the red group shown on the right statistical parametric map. DLB, dementia with Lewy bodies; FTLD, frontotemporal lobar degeneration; FWE, familywise error rate; L, left; R, right; UNC, uncorrected (for multiple comparisons).

matter volume in the region of the precentral and postcentral gyri. Similar findings have been reported in Parkinson's disease, suggesting that atrophy in this region may be a feature of Lewy body pathology more generally. ${ }^{28}$ The DLB group also demonstrated occipital lobe volume loss, in keeping with previous reports of occipital hypometabolism revealed using fluorodeoxyglucose-positron emission tomography. ${ }^{23} 29$ While primary motor, sensory and occipital cortex changes are often considered relatively spared in most degenerative dementias, these results suggest that there may be subtle but detectable changes in these regions in DLB; this, in turn, provides clear hypotheses that can be tested using more advanced MRI techniques.

There was considerable overlap in the pattern of frontal lobe atrophy between the FTLD-tau groups and the TDP43A group, ${ }^{30}$ particularly in the ventrolateral prefrontal cortex. However, the TDP43A pathology group appeared to have a more symmetrical presentation with greater parietal extension, while the left hemisphere was more affected in the 3-repeat-tau and 4-repeat-tau groups. The TDP43C group, which predominantly manifests clinically as semantic dementia, had extensive left anterior temporal lobe atrophy as previously reported in other studies. 3233

Although this study benefits from an unprecedented sample size of ante-mortem imaging acquired in patients with pathological confirmation of their diagnosis, analysis using the most advanced SPM software and, where possible, strict statistical thresholding, it has a number of limitations. Including data from multiple sites increases the sample size, the power to detect statistical differences between groups and importantly the generalizability of the findings, but it also introduces potential confounds relating to the different MRI scanners and pulse sequences used to acquire the images. To account for this, magnetic field strength, which is likely to be the most significant source of variability, ${ }^{34}$ was included along with acquisition site as factors in the model. Although the overall sample size is larger than those previously reported in the literature, sample sizes were insufficient to stratify beyond the primary pathological diagnosis, noting that in practice many patients will have multiple neurodegenerative pathologies of varying extent and severity, and concomitant vascular disease, all of which may influence developing patterns of brain volume loss. ${ }^{35} 36$ In addition, control subjects were not pathologically confirmed; therefore, we cannot rule out presymptomatic pathology in this group, which may reduce the power in comparing the pathology groups with controls. However, this does not affect the more clinically relevant between-pathology group comparisons. Although not quite reaching statistical significance $(p=0.05)$, there was a lower proportion of men included in the younger control group 


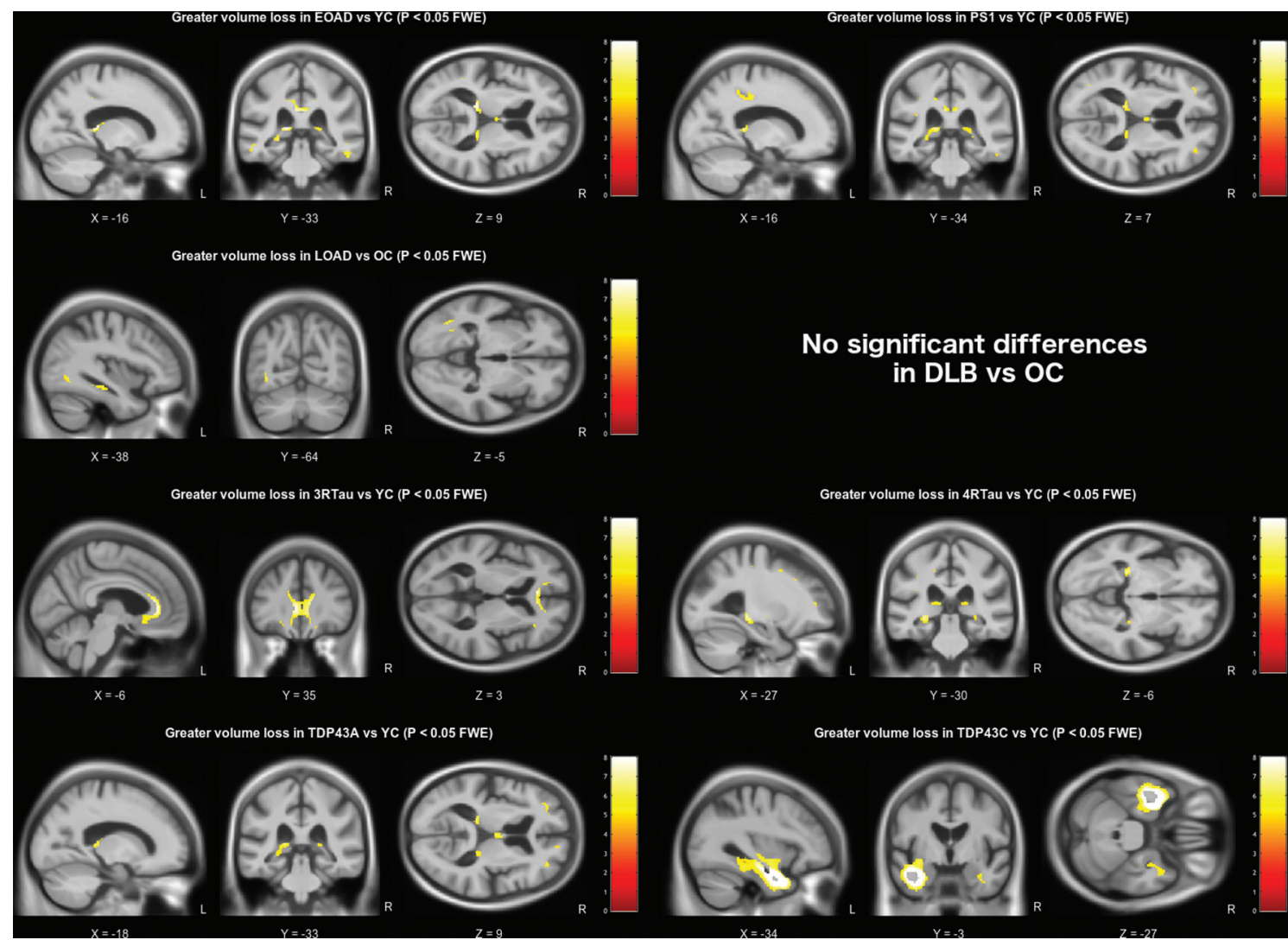

Figure 5 White matter volume differences based on pairwise comparison with the appropriate age-matched control group. All results are shown at a familywise error corrected significance level of $p<0.05$. Brain slices are displayed based on peak voxel location. DLB, dementia with Lewy bodies; EOAD, early-onset Alzheimer's disease; FTLD, frontotemporal lobar degeneration; FWE, familywise error rate; L, left; LOAD, late-onset Alzheimer's disease; OC, older controls; PS1, presenilin-1; R, right; YC, younger controls.

than the other groups; however, as all subjects were included in a single model, sex differences are adjusted for using precise estimates from the total number of men and women included in the model. Finally, as with many research studies, there may be selection bias in the patients included in this cohort: those patients with memory and language led presentations, and those with familial forms of dementia, are more likely to take part in research, including brain donation, than patients with more challenging behavioural problems, for example. This may affect the representation of pathologies in this cohort and reduce how well the results generalise to the wider population.

Although the advent of molecular imaging provides a direct means of identifying underlying pathology during life, ${ }^{37} 38$ the ability to use information from widely available, routinely acquired, noninvasive MRI scans is currently much more applicable in clinical practice; and structural MRI continues to be the most widely used surrogate marker of neurodegeneration, reflected by its inclusion in a recently proposed classification scheme, ${ }^{39}$ and its continued inclusion in research diagnostic guidelines. ${ }^{40}$ Ultimately, however, it is only by using robust methodologies to compare in vivo tests with post-mortem confirmation in large and varied data sets that the diagnostic utility of any dementia biomarker can be assessed. The data from this study-which have been uploaded to the NeuroVault repository for independent scrutiny-provide much needed validation of the regions of the brain where neurodegenerative pathologies are detectable during life, and as such may have greater utility in clinical decision making. In addition, this study highlights where evidence is lacking and demonstrates that voxelwise brain mapping techniques applied to well-defined groups can reveal potentially overlooked regions that warrant further investigation as imaging biomarkers.

\section{Author affiliations}

${ }^{1}$ Dementia Research Centre, University College London Institute of Neurology, London, UK

${ }^{2}$ Alzheimer Centre, VU University Medical Centre, Amsterdam, The Netherlands ${ }^{3}$ Institute for Ageing and Health, Newcastle University, Newcastle upon Tyne, UK ${ }^{4}$ Department of Radiology and Nuclear Medicine, VU Medical Center, MS Center, Amsterdam, The Netherlands

${ }^{5}$ Department of Brain Repair and Rehabilitation, University College London Institute of Neurology, London, UK

${ }^{6}$ Department of Medical Physics \& Biomedical Engineering, University College London Faculty of Engineering Sciences, London, UK

${ }^{7}$ Department of Psychiatry, University of Cambridge, Cambridge, UK

${ }^{8}$ FMRIB Centre, Nuffield Department of Clinical Neurosciences, University of Oxford, Oxford, UK

${ }^{9}$ Wellcome Trust Centre for Neuroimaging, UCL Institute of Neurology, London, UK

Acknowledgements Funding The Dementia Research Centre is an Alzheimer's Research UK coordinating centre. The authors acknowledge the support of Alzheimer's Research UK (grant number ART-NCG2010B-2), the Medical Research Council (grant number MR/J014257/2), the NIHR Queen Square Dementia Biomedical Research Unit, UCL/H Biomedical Research Centre, the Leonard Wolfson Experimental Neurology Centre, the NIHR Newcastle Biomedical Research Unit in Lewy body dementia and the NIHR Cambridge Biomedical Research Unit in Dementia. LH is supported by funding from Alzheimer's Research UK and a UCL Impact Studentship. NCF and JTOB hold NIHR Senior Investigator Awards. JMS acknowledges the support of the NIHR Queen Square Dementia BRU, the NIHR UCL/H Biomedical Research Centre, Wolfson Foundation, EPSRC (EP/J020990/1), MRC (CSUB19166), ARUK (ARUK-Network 2012-6-ICE; ARUK-PG2014-1946) and European Commission (H2020-PHC-2014-2015-666992).

Contributors $L H, G R R$ and JMS designed the study and wrote the manuscript. $L H$ performed the analysis. FB, EJB, FB, PS, JTOB and NCF collected data and revised the manuscript.

Competing interests None declared. 
Ethics approval National Research Ethics Service Committee London South East.

Provenance and peer review Commissioned; externally peer reviewed.

Data sharing statement To request access to the underlying research materials, please contact the corresponding author.

Open Access This is an Open Access article distributed in accordance with the terms of the Creative Commons Attribution (CC BY 4.0) license, which permits others to distribute, remix, adapt and build upon this work, for commercial use, provided the original work is properly cited. See: http://creativecommons.org/ licenses/by/4.0/

(c) Article author(s) (or their employer(s) unless otherwise stated in the text of the article) 2017. All rights reserved. No commercial use is permitted unless otherwise expressly granted.

\section{REFERENCES}

1 Jack CR, Knopman DS, Jagust WJ, et al. Tracking pathophysiological processes in Alzheimer's disease: an updated hypothetical model of dynamic biomarkers. Lancet Neurol 2013;12:207-16

2 Jack CR, Petersen RC, Xu YC, et al. Medial temporal atrophy on MRI in normal aging and very mild Alzheimer's disease. Neurology 1997;49:786-94.

3 Fox NC, Warrington EK, Rossor MN. Serial magnetic resonance imaging of cerebral atrophy in preclinical Alzheimer's disease. The Lancet 1999:353:2125.

4 Sperling RA, Aisen PS, Beckett LA, et al. Toward defining the preclinical stages of Alzheimer's disease: recommendations from the National Institute on AgingAlzheimer's Association workgroups on diagnostic guidelines for Alzheimer's disease. Alzheimers Dement 2011:7:280-92.

5 Albert MS, DeKosky ST, Dickson D, et al. The diagnosis of mild cognitive impairment due to Alzheimer's disease: recommendations from the National Institute on AgingAlzheimer's Association workgroups on diagnostic guidelines for Alzheimer's disease. Alzheimers Dement 2011;7:270-9.

6 McKhann GM, Knopman DS, Chertkow H, et al. The diagnosis of dementia due to Alzheimer's disease: recommendations from the National Institute on AgingAlzheimer's Association workgroups on diagnostic guidelines for Alzheimer's disease Alzheimers Dement 2011;7:263-9.

7 Rascovsky K, Hodges JR, Knopman D, et al. Sensitivity of revised diagnostic criteria for the behavioural variant of frontotemporal dementia. Brain 2011;134(Pt 9):2456-77.

8 Gorno-Tempini ML, Hillis AE, Weintraub S, et al. Classification of primary progressive aphasia and its variants. Neurology 2011;76:1006-14.

9 Cash DM, Rohrer JD, Ryan NS, et al. Imaging endpoints for clinical trials in Alzheimer's disease. Alzheimers Res Ther 2014;6:87.

10 Barkhof F, Fox NC, Bastos-Leite AJ, et al. Neuroimaging in dementia [Internet]. Berlin, Heidelberg: Springer Berlin Heidelberg, 2011. Available from. http://link.springer.com/ (accessed 21 Mar 2016).

11 Harper L, Barkhof F, Scheltens P, et al. An algorithmic approach to structural imaging in dementia. J Neurol Neurosurg Psychiatry 2014;85:692-8.

12 Beach TG, Monsell SE, Phillips LE, et al. Accuracy of the clinical diagnosis of Alzheimer disease at national institute on aging Alzheimer disease centers, 2005-2010. J Neuropathol Exp Neurol 2012;71:266-73.

13 Harper L, Fumagalli GG, Barkhof F, et al. MRI visual rating scales in the diagnosis of dementia: evaluation in 184 post-mortem confirmed cases. Brain 2016:139:1211-25.

14 Ashburner J, Friston KJ. Unified segmentation. Neuroimage 2005;26:839-51.

15 Ashburner J. A fast diffeomorphic image registration algorithm. Neuroimage 2007:38:95-113.

16 Barnes J, Ridgway GR, Bartlett J, et al. Head size, age and gender adjustment in MRI studies: a necessary nuisance? Neuroimage 2010:53:1244-55.
17 Koedam EL, Lauffer V, van der Vlies AE, et al. Early-versus late-onset Alzheimer's disease: more than age alone. J Alzheimers Dis 2010;19:1401-8.

18 Ryan NS, Keihaninejad S, Shakespeare TJ, et al. Magnetic resonance imaging evidence for presymptomatic change in thalamus and caudate in familial Alzheimer's disease. Brain 2013;136:1399-414.

19 Frisoni GB, Pievani M, Testa C, et al. The topography of grey matter involvement in early and late onset Alzheimer's disease. Brain 2007;130:720-30.

20 Greicius MD, Srivastava G, Reiss AL, et al. Default-mode network activity distinguishes Alzheimer's disease from healthy aging: evidence from functional MRI. Proc Natl Acad Sci U SA 2004;101:4637-42.

21 Aggleton JP, Pralus A, Nelson AJ, et al. Thalamic pathology and memory loss in early Alzheimer's disease: moving the focus from the medial temporal lobe to Papez circuit. Brain 2016:139:1877-90.

22 Cash DM, Ridgway GR, Liang Y, et al; Dominantly Inherited Alzheimer Network (DIAN). The pattern of atrophy in familial Alzheimer disease: volumetric MRI results from the DIAN study. Neurology 2013;81:1425-33.

23 Kantarci K, Lowe VJ, Boeve BF, et al. Multimodality imaging characteristics of dementia with Lewy bodies. Neurobiol Aging 2012;33:2091-105.

24 Nedelska Z, Ferman TJ, Boeve BF, et al. Pattern of brain atrophy rates in autopsyconfirmed dementia with Lewy bodies. Neurobio/ Aging 2015;36:452-61.

25 Burton EJ, Barber R, Mukaetova-Ladinska EB, et al. Medial temporal lobe atrophy on MRI differentiates Alzheimer's disease from dementia with Lewy bodies and vascular cognitive impairment: a prospective study with pathological verification of diagnosis. Brain 2009:132(Pt 1):195-203

26 Vemuri P, Simon G, Kantarci K, et al. Antemortem differential diagnosis of dementia pathology using structural MRI: differential-stand. Neuroimage 2011:55:522-31.

27 Whitwell JL, Josephs KA, Murray ME, et al. MRI correlates of neurofibrillary tangle pathology at autopsy: a voxel-based morphometry study. Neurology 2008;71:743-9.

28 Rosenberg-Katz K, Herman T, Jacob Y, et al. Gray matter atrophy distinguishes between Parkinson disease motor subtypes. Neurology 2013;80:1476-84.

29 Toledo JB, Cairns NJ, Da X, et al; Alzheimer's Disease Neuroimaging Initiative (ADNI). Clinical and multimodal biomarker correlates of ADNI neuropathological findings. Acta Neuropathol Commun 2013;1:65.

30 Whitwell JL, Warren JD, Josephs KA, et al. Voxel-based morphometry in tau-positive and tau-negative frontotemporal lobar degenerations. Neurodegener Dis 2004;1(45):225-30

31 Grossman M, Libon DJ, Forman MS, et al. Distinct antemortem profiles in patients with pathologically defined frontotemporal dementia. Arch Neurol 2007;64:1601-9.

32 Rohrer JD, Geser F, Zhou J, et al. TDP-43 subtypes are associated with distinct atrophy patterns in frontotemporal dementia. Neurology 2010;75:2204-11.

33 Whitwell JL, Jack CR, Parisi JE, et al. Does TDP-43 type confer a distinct pattern of atrophy in frontotemporal lobar degeneration? Neurology 2010;75:2212-20.

34 Han X, Jovicich J, Salat D, et al. Reliability of MRI-derived measurements of human cerebral cortical thickness: the effects of field strength, scanner upgrade and manufacturer. Neuroimage 2006;32:180-94.

35 Attems J, Jellinger K. Neuropathological correlates of cerebral multimorbidity. Curr Alzheimer Res 2013;10:569-77.

36 White LR, Edland SD, Hemmy LS, et al. Neuropathologic comorbidity and cognitive impairment in the nun and Honolulu-Asia aging studies. Neurology 2016;86:1000-8.

37 Rowe CC, Villemagne VL. Amyloid imaging with PET in early Alzheimer disease diagnosis. Med Clin North Am 2013;97:377-98.

38 Ossenkoppele R, Schonhaut DR, Schöll M, et al. Tau PET patterns mirror clinical and neuroanatomical variability in Alzheimer's disease. Brain 2016;139:1551-67.

39 Jack CR, Bennett DA, Blennow K, et al. A/T/N: an unbiased descriptive classification scheme for Alzheimer disease biomarkers. Neurology 2016;87:539-47.

40 Dubois B, Feldman HH, Jacova C, et al. Advancing research diagnostic criteria for Alzheimer's disease: the IWG-2 criteria. Lancet Neurol 2014:13:614-29. 